\title{
BMJ Open Quality Implementing a three-hourly feeding schedule in stable preterm infants to decrease maternal fatigue
}

\author{
Abhishek S Aradhya, Inderjot Kaur, Rima Gupta, Sonaljot Kaur, \\ Yamasandi Siddegowda Shrimanth (D) , Parveen Darshan Masih, \\ Praveen Kumar (1)
}

To cite: Aradhya AS, Kaur I, Gupta R, et al. Implementing a three-hourly feeding schedule in stable preterm infants to decrease maternal fatigue. BMJ Open Quality 2021;10:e001439. doi:10.1136/ bmjoq-2021-001439

Received 26 February 2021 Accepted 30 May 2021
Check for updates

(C) Author(s) (or their employer(s)) 2021. Re-use permitted under CC BY-NC. No commercial re-use. See rights and permissions. Published by BMJ.

Department of Pediatrics, Post Graduate Institute of Medical Education and Research, Chandigarh, India

Correspondence to Dr Praveen Kumar; drpkumarpgi@gmail.com

\section{ABSTRACT}

Background A three-hourly feeding schedule has been shown to be as safe as a two-hourly schedule in preterm neonates. It saves nursing time and may be less tiring for the mothers. However, tradition and apprehensions have prevented its wider acceptance. We used a quality improvement approach to implement a three-hourly feeding schedule in stable preterm infants $>32$ weeks postmenstrual age (PMA) in our unit through a series of plan-do-study-act (PDSA) cycles.

Methods All preterm neonates $>32$ weeks PMA, who were on full enteral feeds and without any respiratory support were eligible. The key quantitative outcome was maternal fatigue score. Safety was assessed in terms of episodes of hypoglycaemia and feed intolerance. Qualitative experiences from nursing staff were captured. The volume of expressed breastmilk and requirement of formula feeds were also recorded. After recording baseline data on a two-hourly feeding schedule, four PDSA cycles were sequentially completed over 21 weeks. The results of each PDSA cycle informed the change strategy for the next cycle.

Results In the baseline phase, five neonates on a two-hourly schedule were studied. In PDSA cycles I, II, III and IV, a cumulative of 122 neonates were studied on a three-hourly schedule. There was a significant decrease in median maternal fatigue score (13 (IQR 8-23) to 3 (IQR 1-6); $p=0.01)$ ). Only one neonate had feed intolerance, while two had mild asymptomatic transient hypoglycaemia. Six (5\%) neonates were shifted to twohourly feeds temporarily due to transient reasons. Nursing staff felt mothers could devote more time to Kangaroo mother care. The volume of expressed breastmilk and requirement of formula feeds were not different from the three-hourly schedule.

Conclusions It was possible to change the traditional two-hourly feeding schedule to three-hourly in stable preterm infants using a quality improvement approach, while objectively documenting its safety and benefits.

\section{INTRODUCTION}

Full term and late preterm infants are breastfed as per their demand. Feeding of preterm neonates should also ideally be cuebased, but identification of feeding cues in preterm neonates needs careful observation and expertise, and is not practised routinely.
Preterm infants less than 34-week gestation are usually fed expressed breastmilk (EBM) by gavage or spoon, at arbitrarily fixed intervals, usually every two-hourly. A lot of research in relation to initiation and advancement of feeds in preterm infants is available, but there are few studies addressing the issue of feeding intervals. $^{1-7}$

\section{PROBLEM}

A two-hourly schedule is quite tiring for the mothers and leaves little continuous time for Kangaroo mother care (KMC). Mothers recovering from pregnancy and delivery find it hard to cope with it. They need to interrupt KMC for expression of milk and feeding. ${ }^{8} 9$ KMC involves providing early, prolonged and continuous skin to skin contact for low birthweight babies by their mothers. It is currently a standard of care for preterm neonates and has been shown to decrease mortality by giving warmth, improving breastmilk feeding and optimising nutrition, providing neurosensory stimulation and preventing nosocomial infections. A two-hourly schedule also increases the total nursing time spent on feeding, which can be challenging, especially in units with lower nurse to patient ratios. This affects the ability of nurses to complete other essential clinical care activities.

\section{CONTEXT}

Postgraduate Institute of Medical Education and Research, Chandigarh is a tertiary care government hospital in north India. About 30 stable preterm neonates are under care each day, spread across three locations-transitional nursery (TN), low birthweight (LBW) room and neonatal nursery (NNN) (table 1 ). Mothers express their milk 2-3 hourly as per prescribed feeding schedule and mother's own milk is fed to the babies. There is no facility for human milk bank or prolonged 
Table 1 Study settings

\begin{tabular}{|c|c|c|}
\hline Area & $\begin{array}{l}\text { No. of beds } \\
\text { (baby:bed ratio) }\end{array}$ & Characteristics \\
\hline Transitional nursery (TN) & $\begin{array}{l}5 \text { (variable, but } \\
\text { usually }>1: 1 \text { ) }\end{array}$ & $\begin{array}{l}\text { 31-37 weeks, nursed under radiant warmers, awaiting transfer to other areas, } \\
\text { pending availability of bed. Infants are expected to stay here only for few hours, } \\
\text { but not uncommonly have to stay longer due to non-availability of bed in other } \\
\text { areas }\end{array}$ \\
\hline $\begin{array}{l}\text { Low birth weight (LBW) } \\
\text { room }\end{array}$ & $6(1: 1)$ & 34-37 weeks, $1.4-1.8 \mathrm{~kg}$ neonates nursed with mother \\
\hline
\end{tabular}

NICU, Neonatal Intensive Care Unit.

storage of breast milk. Expression of breastmilk and $\mathrm{KMC}$ is encouraged for all preterm infants. Mothers have 24 hours access and are involved in baby-care activities. If the EBM is insufficient, formula milk is used to meet the deficit. The infants are fed by gavage till a postmenstrual age (PMA) of 30-32 weeks, after which they are offered spoon or Paladai (small cup-like utensil with narrow tip) feeds as per individual assessment. Bottle feeding is not used in our unit.

\section{AVAILABLE KNOWLEDGE AND RATIONALE}

A three-hourly feeding schedule can overcome the problems of both mothers and nurses, and potentially offer an advantage of faster feed advancement. ${ }^{10}$ Physiological studies indicate that increasing the feeding interval to 3 hours may lead to better gastric emptying and less risk of feed intolerance. ${ }^{12}$ However, three-hourly feeding regimes are not commonly practised in preterm infants, due to concerns about hypoglycaemia and feed intolerance. In randomised controlled trials (RCTs) of twohourly versus three-hourly feeding schedules, the risk of hypoglycaemia, feed intolerance and necrotising enterocolitis has been similar. ${ }^{56}$ While Dhingra et al showed decreased use of nursing time, Ibrahim et al found earlier birthweight attainment with three-hourly schedules. ${ }^{56}$ Both trials enrolled neonates within 12-48 hours of life and found the incidence of feed intolerance and hypoglycaemia to be similar between the two schedules. Apart from individual concerns, there may be many barriers to the implementation of evidence into clinical practice. ${ }^{11}$ Prevailing beliefs and lack of clear written protocols also prevent changing already established practices. ${ }^{12} 13$ With evidence of safety and other patient benefits of a three-hourly feeding schedule from controlled trials, we planned to introduce the three-hourly schedule in our unit in a gradual manner using plan-do-study-act (PDSA) cycles.

\section{AIM}

We aimed to decrease maternal fatigue by implementing a three-hourly feeding schedule in stable preterm infants
$>24$ hours of life with PMA >32 weeks and weighing $>1000 \mathrm{~g}$ at birth, over a period of 4 months.

\section{METHODS}

Design

We formed a multidisciplinary Quality improvement (QI) team comprising of an academic consultant, senior resident physician, junior resident physician and nursing officers from abovementioned three neonatal care areas of the hospital. One nursing officer from each area was given the responsibility of supervising the implementation in their area, data collection and attending team meetings. Three nursing officers together with a senior resident and a consultant led the QI initiative for the entire duration. We decided to address the barriers for implementation through a series of PDSA cycles.

\section{Ethical considerations}

The mothers were explained about the study in their own language and verbal consent was obtained. We implemented evidence-based strategies at the system level and objectively monitored for any safety issues. The treating physicians and nurses had complete independence for any management decisions regarding individual patients.

\section{Patient involvement}

Patients were not directly involved in the design or implementation.

\section{Measurements}

We aimed to decrease the maternal fatigue scores by $20 \%$ by implementing a three-hourly feeding schedule. Maternal fatigue scores were objectively assessed using a modified fatigue symptom checklist, a published and validated questionnaire. ${ }^{14}$ Stable preterm infant was defined as a neonate between $32^{1 / 7}$ and $36^{6 / 7}$ weeks PMA and, not on intravenous fluids or respiratory support. We excluded infants with gastro-oesophageal reflux (GER) and major congenital malformations. The compliance to the new schedule was assessed by recording the actual number of neonates getting three-hourly feeds out of all those eligible. We assessed safety by tracking the number of neonates developing hypoglycaemia and the number 
of episodes of feed intolerance. Blood glucose was monitored as per routine protocol for all preterm neonates in the first 72 hours of life and as per clinical indications in the later period. Descriptive statistics were used to describe the baseline variables. Numerical variables were tested for normality by Kolmogorov-Smirnov test. Normally distributed variables were compared by unpaired t-test after evaluating equality of variance by Levene's test ( $\mathrm{F}$ test). Variables with skewed distribution were analysed with non-parametric Mann-Whitney $\mathrm{U}$ test. $\mathrm{P}$ value of less than 0.05 was taken as significant. The analysis was done using statistical software packages IBM-SPSS V.23 (SPSS). Run charts were used to display the serial measurements of compliance to the three-hourly schedule. We followed the SQUIRE 2.0 guidelines for reporting. ${ }^{15}$

\section{Strategy}

During the baseline period of 11 days, the team tracked all neonates with PMA $>32$ weeks and weighing $>1000 \mathrm{~g}$, who were staying in the $\mathrm{TN}$ for more than 24 hours. In this period, five such neonates, on usual two-hourly feeds, were monitored during their stay in TN initially and later in LBW room until discharge. Nursing officers recorded maternal fatigue scores after 5-7 days of the infant being on the feeding schedule. After the baseline period, we implemented the three-hourly feeding schedule in these areas sequentially, in four PDSA cycles. During each cycle, maternal fatigue scores were recorded after 5-7 days of initiating the three-hourly feeds. The results of each PDSA informed the change strategy for the next cycle.

\section{PDSA cycles}

\section{PDSA cycle \# I}

In PDSA cycle I (2-10 July 2016), we shared the existing evidence on three-hourly feeding schedule with the resident doctors and nursing officers of all three areas in a discussion forum. As the resident doctors and nursing officers were apprehensive, we decided to first test the feasibility and safety of the new schedule in relatively larger neonates and in more stable areas like TN and LBW room. We wrote a draft protocol for three-hourly feeding in neonates $>32$ weeks PMA and $>1250 \mathrm{~g}$ birth weight who were on full enteral feeds and no respiratory support. The draft protocol was approved by the area consultant and the information was disseminated through posters and whatsapp messages.

\section{PDSA cycle \# II}

After gaining some confidence from the first PDSA cycle, we extended the new schedule to $\mathrm{NNN}$ - a relatively less stable area, and to infants with birth weight $>1000 \mathrm{~g}(13-28$ July 2016). The nursing officers from TN and LBW room, where the schedule was tested in PDSA 1, shared their experiences with the nursing officers of NNN. The data from the first test cycle encouraged the nursing officers and resident doctors of NNN to try out the three-hourly feeding schedule.

During PDSA cycle II, we found that in NNN, which had a mixed population of stable as well as relatively sicker infants, some neonates were on two-hourly schedules due to oxygen support or GER, while some were on three-hourly schedules. For example, during a 6-hour period, feeding was scheduled at 2nd, 4th and 6th hour for infants on respiratory assistance or GER, whereas it was scheduled at 3rd and 6th hour for the stable ones. This led to difficulties in organising and clustering of nursing activities, and confusions in scheduling. During the feeding process, nurses have to help mothers with expression of milk, or prepare fresh formula milk, and sterilise the katories (Katori is a small steel bowl of around $50 \mathrm{~mL}$, in which the mother expresses her breast milk).

\begin{tabular}{|c|c|c|c|c|c|}
\hline Phase & Baseline & PDSA I & PDSA II & PDSA III & PDSA IV \\
\hline Duration (dates) & 17.6 .16 to 28.6 .16 & 2.7.16 to 10.7 .16 & 13.7 .16 to 28.7 .16 & 4.8.16 to 17.8 .16 & 12.10 .16 to 28.10 .16 \\
\hline Care area & $\begin{array}{l}\text { TN } \\
\text { LBW }\end{array}$ & $\begin{array}{l}\text { TN } \\
\text { LBW }\end{array}$ & $\begin{array}{l}\text { LBW } \\
\text { NNN }\end{array}$ & $\begin{array}{l}\text { TN } \\
\text { LBW } \\
\text { NNN }\end{array}$ & $\begin{array}{l}\text { TN } \\
\text { NNN }\end{array}$ \\
\hline $\begin{array}{l}\text { Mean gestation at birth in weeks } \\
\text { (range) }\end{array}$ & $33(32-36)$ & $33(32-35)$ & $32(28-37)$ & $33(28-37)$ & $31(28-36)$ \\
\hline Mean birth weight in g (range) & $1587(1437-1677)$ & $1593(1400-1850)$ & $1524(1020-2200)$ & $1522(848-2383)$ & $1358(800-2148)$ \\
\hline Mean PMA at enrolment (range) & $33(32-36)$ & $33(32-35)$ & $33(32-37)$ & $34(32-37)$ & $34(32-37)$ \\
\hline Mean weight at enrolment in $\mathrm{g}$ (range) & $1587(1437-1677)$ & $1593(1400-1850)$ & 1569 (1151-2200) & $1543(1140-2380)$ & $1598(1080-2588)$ \\
\hline
\end{tabular}

The details and results of the PDSA cycles are shown in table 3 .

LBW, low birthweight; NICU, Neonatal Intensive Care Unit; NNN, neonatal nursery; PDSA, plan-do-study-act; PMA, postmenstrual age; TN, transitional nursery. 


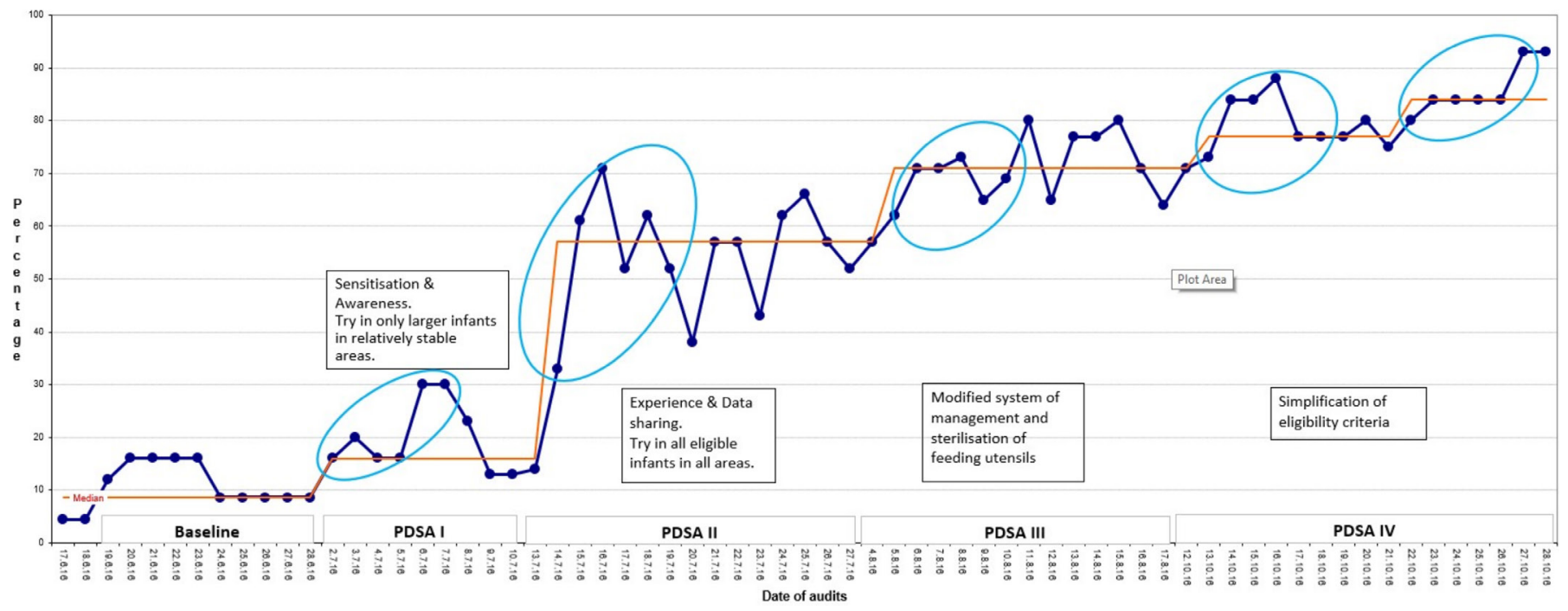

Figure 1 Run chart showing proportion of eligible preterm neonates receiving three-hourly feeds over time. The blue ovals depict significant 'shifts' (six or more data points on same side of median). PDSA, plan-do-study-act.

\section{PDSA cycle \# III}

We modified the system of managing katories in PDSA III (4-17 August 2016). We increased the number of Katoris in circulation from 20 to 50 . This allowed us to reschedule the sterilisation activity to every 6 hours instead of every 2 hours. We also drafted a simple questionnaire to record the qualitative experiences of nurses. The questions pertained to milk expression, feed tolerance, their feelings about the change and any problems they were encountering with the change in the feeding regimen. As some nurses expressed concerns whether the mothers will be able to express the increased volumes required for three-hourly feeding, we decided to assess the volume of expressed breastmilk in a small subset of mothers.

During PDSA III, we observed that there was a confusion regarding the eligibility criteria for three-hourly feeding in NNN, the relatively sicker area. Neonates who

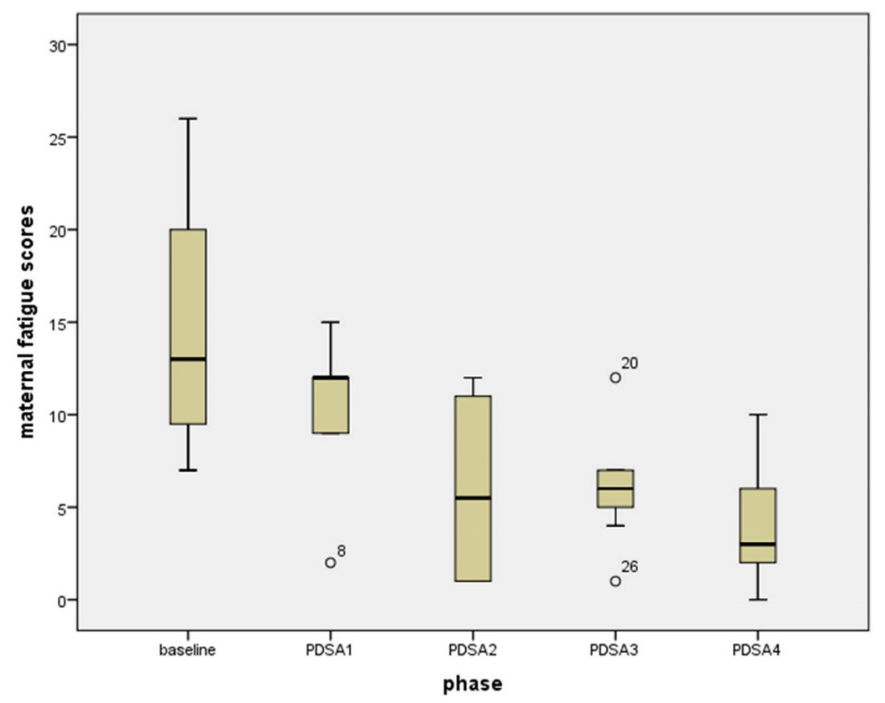

Figure 2 Box-plot of maternal fatigue scores during various PDSA cycles. PDSA, plan-do-study-act. were transferred in from Neonatal Intensive Care Unit (NICU) to NNN, after a difficult course like prolonged ventilation or multisystem problems or lower gestation at birth, were left out of three-hourly schedule due to apprehensions, whereas those with lesser degree of sickness in the initial period were prescribed three-hourly feeds.

\section{PDSA cycle \# IV}

We addressed the issue of selection of eligible babies in PDSA IV (12-24 October 2016). We updated the standard operating protocol to administer three-hourly feeding to all neonates $>32$ weeks PMA and birth weight $>1000 \mathrm{~g}$, with no GER, and no respiratory support irrespective of their previous morbidities. The benefits of three-hourly feeding with respect to less maternal fatigue and safety data from previous PDSA cycles were presented in a group discussion.

The four PDSA cycles were sequentially completed over 21 weeks. The updated data of eligible babies receiving three-hourly feeds and maternal fatigue scores were displayed in all three areas every week.

\section{RESULTS}

A cumulative of 122 neonates were studied on threehourly schedule. Of these, $20(16 \%)$ were on gavage feeds while rest all were on Katori-spoon or Paladai feeds. The demographic features of enrolled neonates in different PDSA cycles are depicted in table 2.

Figure 1 shows the improvement in compliance to the three-hourly feeding schedule over time.

Maternal fatigue scores assessed after 5-7 days of implementation of three-hourly schedule showed a significant reduction as compared with the two-hourly schedule (figure 2). Overall, 6/122 (5\%) neonates were switched to two-hourly feeds due to reasons shown in table 3 . Of these, two neonates had mild asymptomatic transient 
Table 3 Details of the PDSA cycles

\begin{tabular}{|c|c|c|c|c|}
\hline PDSA & $I(n=13)$ & II $(n=33)$ & III $(n=47)$ & IV $(n=29)$ \\
\hline When & 2.7.16 to 10.7 .16 & 13.7.16 to 28.7 .16 & 4.8.16 to 17.8 .16 & 12.10 .16 to 28.10 .16 \\
\hline Plan & $\begin{array}{l}\text { Assess feasibility and } \\
\text { safety of three-hourly } \\
\text { feeding in two areas: TN } \\
\text { and LBW room }\end{array}$ & $\begin{array}{l}\text { Extend the test to } \\
\text { neonates with birth weight } \\
>1000 \mathrm{~g} \text { and to relatively } \\
\text { sicker area (NNN) apart } \\
\text { from TN and LBW room }\end{array}$ & $\begin{array}{l}\text { New system of managing } \\
\text { Katoris } \\
\text { Qualitative experience of } \\
\text { nurses } \\
\text { Assess expressed } \\
\text { breastmilk volume in a } \\
\text { subset of women }\end{array}$ & $\begin{array}{l}\text { Simplify eligibility criteria and } \\
\text { update standard operating } \\
\text { protocol }\end{array}$ \\
\hline
\end{tabular}

$\begin{array}{ll}\text { Do } & \text { Try three-hourly schedule } \\ \text { in infants }>32 \text { weeks } \\ \text { PMA and }>1250 \mathrm{~g} \text { birth } \\ \text { weight; } \\ \text { Track compliance, } \\ \text { hypoglycaemic and feed } \\ \text { intolerance; Record } \\ \text { maternal fatigue scores }\end{array}$

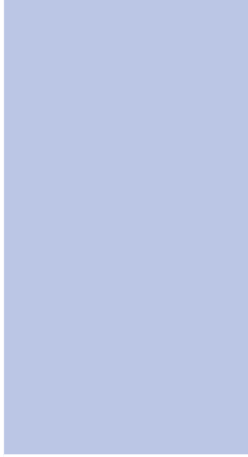

Study
Compliance $16 \%$. Safe

in $13 / 13(100 \%)$

Maternal fatigue scores $12(5-13))$ compared with to stoppage of caffeine). baseline (13 (8-23)) 32/33 (97\%)

Feed intolerance in 1
Three-hourly feeding in TN, LBW room and NNN, in infants $>32$ weeks PMA and $>1000 \mathrm{~g}$ birth weight Track compliance, hypoglycaemia and feed intolerance; Record maternal fatigue scores
Record qualitative

Compliance 57\%. Safe in Compliance $71 \%$. to perceived excessive weight loss (not confirmed on chart review).

Maternal fatigue scores lower- median (IQR) 4 (1-8)

Difficulties encountered in clustering of nursing activities in NNN due to some sicker babies on two-hourly feeds and others on three-hourly feeds

$\begin{array}{lll}\text { Act } & \text { Extend the new feeding } & \text { Reorganise the nursing } \\ \text { schedule to infants }>32 & \text { activities related to } \\ \text { weeks PMA and }>1000 \mathrm{~g} & \text { sterilisation of Katoris } \\ \text { birth weight and in NNN } & \text {. Obtain qualitative } \\ & \text { feedback from nurses }\end{array}$

Address the confusion in eligibility criteria and continue with new sterilisation schedule

\section{Continue three-hourly} feeding in all three care areas in infants $>32$ weeks PMA and $>1000 \mathrm{~g}$ birth weight Increase Katoris in circulation from 20 to 50 , and change sterilisation schedule from two hourly to six-hourly experience of nurses Record volume of expressed breastmilk in seven randomly selected mothers

Track compliance, hypoglycaemia and feed intolerance, maternal fatigue scores Safe in $45 / 47$ (96\%) Mild asymptomatic transient hypoglycaemia in 2 (blood glucose 35 and 36)-switched to twohourly, No feed intolerance Maternal fatigue scores median (IQR) 6 (4-7) Qualitative feedback of

Three-hourly feeding in all babies $>32$ weeks PMA and $>1000 \mathrm{~g}$ birth weight in TN, LBW room and NNN, if currently not on respiratory support or having GER, irrespective of previous morbidities.

Continue tracking compliance, hypoglycaemia and feed intolerance; maternal fatigue scores

\section{Compliance $84 \%$. Safe in 29/29 (100\%)}

No case of hypoglycaemia, Two neonates changed to two-hourly feeds due to suspected GER.

Maternal fatigue scores median (IQR)

3 (1-6)

No problems with the new Katori sterilisation schedule. hourly feeds;

New problem-confusion in eligibility criteria in NNN area due to previous morbidities of neonates transferred in from NICU.
Final written policy and SOP for three-hourly feeding in TN, LBW room and NNN. Periodic monitoring plan for compliance and any unexpected problems

GER, gastro-oesophageal reflux; LBW, low birthweight; NICU, Neonatal Intensive Care Unit; NNN, neonatal nursery; PDSA, plan-do-studyact; PMA, postmenstrual age; TN, transitional nursery. 
hypoglycaemia. There was one case of feed intolerance and two cases of GER (3/122 (2.4\%)). Qualitative experiences of eight randomly selected nursing officers were objectively recorded. All of them favoured three-hourly feeds. After changing from two-hourly to three-hourly schedules, they felt that for the initial 2-3 feeds, it was more challenging for mothers to express the increased volumes required and the neonates took little longer to feed. However, these were very transient and both mothers and neonates adjusted quickly. Nursing time was saved, and they could devote time for better documentation and other nursing-related works. The volume of expressed breastmilk was also measured in seven randomly selected mothers and they could express the required volumes. There was no increase in the requirement of formula feeds.

To ensure sustenance, the key results of the study were displayed as posters in the clinical areas and a written policy was made available. In each clinical area, a QI champion nurse along with a senior resident took the responsibility of supervision and orientation of new staff. Luckily, the rotations/changes in nursing staff are not very frequent in our unit. Formal audits were stopped as the new schedule became part of unit practice. However, informal reviews of feeding charts revealed sustained incorporation of three-hourly feeding in the routine.

\section{LESSONS AND LIMITATIONS}

In scenarios of predominant breastmilk feeding and intimate maternal involvement in baby-care activities like ours, mothers spend 20-30 min expressing their milk and another 20-30 min for the complete process of feeding the child. With a two-hourly feeding schedule, they are left with only about $60-80 \mathrm{~min}$, before the next feed is due. In this duration, they are expected to provide KMC and other baby-care activities, leaving little time for rest. A three-hourly feeding schedule has been shown to consume less nursing time and more time is available to mothers for KMC and for their own rest.

We focused on maternal fatigue as the outcome, after discussion with the nurses, as it is a patient-centred outcome and nurses visualised several benefits for the babies if mothers could get more time. Our unit is scattered across several physically separate care areas. Each area has its own case-mix and unique set of challenges. Hence, we started in a small manner from relatively quiet areas and in relatively larger weight neonates. The sharing of safety data and experiences by the nurses with their colleagues in other care areas built their confidence. This allowed us to expand the test to all care areas and in all eligible infants.

Although maternal fatigue score was the main predecided outcome measure, based on the nursing feedback, we incorporated qualitative questionnaire for nursing experience and maternal breastmilk output measurement in a small subset. This helped to further enhance their confidence and uptake of the three-hourly schedule. We did encounter unanticipated problem in the form of management of feeding utensils and their sterilisation schedule. The solution to this problem was provided by the nurses themselves and was easily implementable with minimal extra costs. The stock of the utensils had to be replenished periodically, however, this was similar to the previous arrangements for the same.

In this initiative, we could not formally involve the mothers in the design and conduct of the project. This is a limitation which can be addressed in future endeavours. There are several other perceived and previously documented benefits of a three-hourly schedule, for example, increased duration of KMC, earlier attainment of birth weight, decreased length of stay and better compliance with other nursing care activities. Many of these benefits have already been observed in RCTs. With our focus being on implementation, we restricted ourselves to measurement of limited number of variables. Another limitation was that although informal reviews of feeding charts revealed sustained incorporation of three-hourly feeding in the routine, formal audits were stopped.

Overall, we learnt that though the switch from a twohourly to three-hourly feeding schedule sounded easy, it required patience and perseverance, and finding solutions to unexpected problems. Regular team meetings, sharing of data and group discussions during the study helped to address individual concerns, plan the next phase and tackle the challenges. Translating evidence into clinical practice needs tackling various barriers through PDSA cycles and thus making the process of implementation smooth. ${ }^{11-13}$

\section{Conclusion}

We were able to change the traditional two-hourly feeding practice to three-hourly feeding in stable preterm infants by focusing on a patient-centred outcome and applying small changes through serial PDSA cycles, while adapting to the unanticipated challenges. This experience will be useful to many similar scenarios especially across the developing world, where the nursing resources are limited but mothers are available for baby-care activities.

Twitter Praveen Kumar @praveenpginicu

Acknowledgements Three-hourly feed QI group: Prabhdeep Kaur, Harminder Kaur, Simerjeet Kaur, Suman Bura, Sankalp Dudeja for administering fatigue scores for mothers and assisting in data collection.

Contributors ASA conceptualised the study, designed the data collection tool, analysed the data, drafted the initial manuscript and approved the final manuscript. IK assisted in designing the study, collected the data, analysed the data and approved the final manuscript. RG, SK, YSS collected the data and approved the final manuscript. PDM contributed to study design, assisted in designing the data collection tool and approved the final manuscript. PK conceptualised and supervised the design of the study, critically reviewed the data analysis and the manuscript, and approved the final manuscript.

Funding The authors have not declared a specific grant for this research from any funding agency in the public, commercial or not-for-profit sectors. Publication of this article is made Open Access with funding from the Nationwide Quality of Care Network.

Competing interests None declared. 
Patient and public involvement Patients and/or the public were not involved in the design, or conduct, or reporting, or dissemination plans of this research.

Patient consent for publication Not required.

Ethics approval The institute ethics committee approved the study and granted waiver of written individual consent.

Provenance and peer review Not commissioned; externally peer reviewed.

Data availability statement All data relevant to the study are included in the article.

Open access This is an open access article distributed in accordance with the Creative Commons Attribution Non Commercial (CC BY-NC 4.0) license, which permits others to distribute, remix, adapt, build upon this work non-commercially, and license their derivative works on different terms, provided the original work is properly cited, appropriate credit is given, any changes made indicated, and the use is non-commercial. See: http://creativecommons.org/licenses/by-nc/4.0/.

ORCID iDs

Yamasandi Siddegowda Shrimanth http://orcid.org/0000-0003-1219-8002

Praveen Kumar http://orcid.org/0000-0003-4742-8787

\section{REFERENCES}

1 Lane AJ, Coombs RC, Evans DH, et al. Effect of feed interval and feed type on splanchnic haemodynamics. Arch Dis Child Fetal Neonatal Ed 1998;79:F49-53.

2 Bodé S, Dreyer M, Greisen G. Gastric emptying and small intestinal transit time in preterm infants: a scintigraphic method. J Pediatr Gastroenterol Nutr 2004;39:378-82.

3 Rüdiger M, Herrmann S, Schmalisch G, et al. Comparison of 2-h versus $3-\mathrm{H}$ enteral feeding in extremely low birth weight infants, commencing after birth. Acta Paediatr 2008;97:764-9.
4 DeMauro SB, Abbasi S, Lorch S. The impact of feeding interval on feeding outcomes in very low birth-weight infants. $J$ Perinatol 2011;31:481-6.

5 Dhingra A, Agrawal SK, Kumar P, et al. A randomised controlled trial of two feeding schedules in neonates weighing $<$ or $=1750 \mathrm{~g}$. J Matern Fetal Neonatal Med 2009;22:198-203.

6 Ibrahim NR, Kheng TH, Nasir A, et al. Two-hourly versus 3-hourly feeding for very low birthweight infants: a randomised controlled trial. Arch Dis Child Fetal Neonatal Ed 2017;102:F225-9.

7 Dutta S, Singh B, Chessell L, et al. Guidelines for feeding very low birth weight infants. Nutrients 2015;7:423-42.

8 Nyqvist KH, Anderson GC, Bergman N, et al. Towards universal kangaroo mother care: recommendations and report from the first European conference and seventh International workshop on kangaroo mother care. Acta Paediatr 2010;99:820-6.

9 Conde-Agudelo A, Díaz-Rossello JL. Kangaroo mother care to reduce morbidity and mortality in low birthweight infants. Cochrane Database Syst Rev 2016:CD002771.

10 Chu E, Freck S, Zhang L, et al. Three-hourly feeding intervals are associated with faster advancement in very preterm infants. Early Hum Dev 2019;131:1-5.

11 Grol R, Grimshaw J. From best evidence to best practice: effective implementation of change in patients' care. Lancet 2003;362:1225-30.

12 Grol R. Successes and failures in the implementation of evidencebased guidelines for clinical practice. Med Care 2001;39:II-46.

13 Wensing M, van der Weijden T, Grol R. Implementing guidelines and innovations in general practice: which interventions are effective? $\mathrm{Br}$ $J$ Gen Pract 1998;48:991-7.

14 Pugh LC, Milligan R, Parks PL, et al. Clinical approaches in the assessment of childbearing fatigue. J Obstet Gynecol Neonatal Nurs 1999;28:74-80.

15 Ogrinc G, Davies L, Goodman D, et al. Squire 2.0 (standards for quality improvement reporting excellence): revised publication guidelines from a detailed consensus process. BMJ Qual Saf 2016;25:986-92. 